ARTICLE

\title{
Reconfigurable radiofrequency filters based on versatile soliton microcombs
}

\author{
Jianqi $\mathrm{Hu}^{1,3}$, Jijun $\mathrm{He}^{2,3}$, Junqiu Liu (1) 2, Arslan S. Raja², Maxim Karpov (1) 2, Anton Lukashchuk2, \\ Tobias J. Kippenberg (iD ${ }^{2 \otimes} \&$ Camille-Sophie Brès (iD ${ }^{1 凶}$
}

The rapidly maturing integrated Kerr microcombs show significant potential for microwave photonics. Yet, state-of-the-art microcomb-based radiofrequency filters have required programmable pulse shapers, which inevitably increase the system cost, footprint, and complexity. Here, by leveraging the smooth spectral envelope of single solitons, we demonstrate microcomb-based radiofrequency filters free from any additional pulse shaping. More importantly, we achieve all-optical reconfiguration of the radiofrequency filters by exploiting the intrinsically rich soliton configurations. Specifically, we harness the perfect soliton crystals to multiply the comb spacing thereby dividing the filter passband frequencies. Also, the versatile spectral interference patterns of two solitons enable wide reconfigurability of filter passband frequencies, according to their relative azimuthal angles within the round-trip. The proposed schemes demand neither an interferometric setup nor another pulse shaper for filter reconfiguration, providing a simplified synthesis of widely reconfigurable microcombbased radiofrequency filters.

\footnotetext{
${ }^{1}$ École Polytechnique Fédérale de Lausanne, Photonic Systems Laboratory (PHOSL), STI-IEL, Station 11, 1015 Lausanne, Switzerland. ${ }^{2}$ École Polytechnique Fédérale de Lausanne, Laboratory of Photonics and Quantum Measurements (LPQM), SB-IPHYS, Station 3, 1015 Lausanne, Switzerland. ${ }^{3}$ These authors contributed equally: Jianqi Hu, Jijun He. ${ }^{\circledR}$ email: tobias.kippenberg@epfl.ch; camille.bres@epfl.ch
} 
$\mathrm{O}$ wing to the ever-maturing photonic integration, radiofrequency (RF) photonic systems and subsystems have been brought to new height ${ }^{1-5}$, in terms of footprint, scalability, and potentially cost-effectiveness. Particularly, RF filtering towards chip-scale is a key enabling function ${ }^{6-17}$. Paradigm demonstrations include the integration of the basic filtering blocks, such as delay lines ${ }^{7}$, optical spectral shaper ${ }^{8}$, programmable mesh topologies ${ }^{9}$, and ring resonators ${ }^{10-12}$, as well as the use of stimulated Brillouin scattering (SBS) in waveguides ${ }^{13}$. Recently, an all-integrated RF photonic filter has been shown in a monolithic platform ${ }^{14}$. Among others, RF filters constructed from tapped delay line (TDL) structures attract great attention. They can be classified into two types ${ }^{18}$, depending on whether the filtering profile is given by the physical path delays ${ }^{9,14}$ or the light source spectra $7,8,15-17,19-22$. While the former approach is straightforward, a multiwavelength source combined with dispersive propagation also functions as a TDL filter. This greatly simplifies the structural complexity of a finite impulse response filter, as only a single dispersive delay line is needed. Nevertheless, the main complexity is then shifted to the multiwavelength source. Electro-optic (EO) combs $8,19,20$ or mode-locked lasers ${ }^{21,22}$ are generally adopted as light sources, which remain expensive and bulky options.

Integrated optical Kerr combs (microcombs) have appeared as an interesting alternative. Microcombs have already been applied not only to filter RF signals ${ }^{15-17}$ but also for various RF photonic processing, such as true-time delay beamforming ${ }^{23}$, RF channelization $^{24}$, and analog computation ${ }^{25}$. The large comb spacing of microcombs also enhances RF filters with broader Nyquist zone (spur-free range), lower latency ${ }^{15}$, and less dispersion-induced fading, as well as larger number counts of equivalent delay lines ${ }^{16}$, unparalleled by other approaches. However, so far all these microcomb-based RF filters have been implemented on either dark pulses $^{15,26}$ or complex soliton crystal states ${ }^{16,17}$. Additional programmable pulse shaping modules are inevitably required to equalize or smooth the comb spectral shape. Thus, the system complexity is significantly increased while the potential for lowcost and high-volume applications is compromised. To date, harnessing the smooth sech ${ }^{2}$ spectral envelope of single soliton ${ }^{27-29}$ or other regulated dissipative Kerr soliton (DKS) states for photonic $\mathrm{RF}$ filtering is yet to be investigated. Because single-soliton microcombs have facilitated a myriad of applications, ranging from $\mathrm{Tb} / \mathrm{s}$ coherent communication ${ }^{30}$, ultrafast and long-distance ranging ${ }^{31,32}$, dual-comb spectroscopy ${ }^{33}$, and astronomical spectrometer calibration ${ }^{34}$, to microwave synthesis ${ }^{35}$, a great benefit for RF filtering can be expected.

In this paper, we demonstrate soliton microcomb-based RF photonic filters without any external pulse shaping. In addition, the synthesized RF filters can be all optically reconfigured through the internal versatile soliton states. Specifically, we trigger, in a deterministic fashion, the perfect soliton crystals (PSCs) to multiply the comb spacing ${ }^{36,37}$, thereby dividing the RF passband frequencies. Moreover, we achieve filter reconfiguration based on versatile two-soliton microcombs (TSMs). The spectral interference of two solitons is functionally equivalent to an interferometric setup, shifting the filter passband frequency via modification of the angle between them. A proof-of-concept filter reconfiguration experiment is also shown using TSM-based RF filters. The internal exploitation of abundant and regulated soliton formats of microresonator effectively bypasses the need of another programmable pulse shaper and interferometric setup for RF filter tuning ${ }^{15,16}$. Thus, the proposed scheme dramatically reduces the system complexity and form factor of microcomb-based RF filters, and are readily applicable to the current radar systems, $5 \mathrm{G}$ wireless, and satellite communications.

\section{Results}

Principle of soliton microcomb-based RF filters. Figure 1a illustrates the conceptual setup for soliton microcomb-based RF filters. First, a telecom C-band continuous wave (CW) laser initiates microcomb generation, where each comb line serves as the RF filter tap. By modulating the RF signals from a vector network analyzer (VNA) on an electro-optic Mach-Zehnder modulator (MZM), the RF signals are broadcast to each microcomb mode. Then, the upconverted signals are propagated through a spool of single-mode fiber (SMF) to acquire incremental delay between filter taps. Finally, the signals are converted back to the RF domain in a fast photodetector (PD). The detailed experimental setup is described in the "Methods". This arrangement exactly corresponds to a TDL filter, where the power of each comb line $p_{k}$ is the tap weight, and the delay is determined by the comb spacing $f_{m}$ and the accumulated dispersion $\phi_{2}=-\beta_{2} L$ (the product of SMF second-order dispersion $\beta_{2}$ and fiber length $L$ ). When the filter tap weights take the $\operatorname{sech}^{2}$ envelope of a singlesoliton comb (case 1), the RF filter response is given as (see Supplementary Note 1):

$$
H\left(f_{\mathrm{RF}}\right) \sim \cos \left(2 \pi^{2} \phi_{2} f_{\mathrm{RF}}^{2}\right) \sum_{n=-\infty}^{\infty} G\left(f_{\mathrm{RF}}-n f_{\mathrm{FSR}}\right),
$$

where $f_{\mathrm{FSR}}$ and $G\left(f_{\mathrm{RF}}\right)$ are, respectively, defined as $1 /\left(2 \pi \phi_{2} f_{m}\right)$ and $2 \frac{T}{T_{0}} \frac{f_{\mathrm{RF}}}{f_{\mathrm{FSR}}} \sinh ^{-1}\left(\frac{T}{T_{0}} \frac{f_{\mathrm{RF}}}{f_{\mathrm{FSR}}}\right)$, with $T=1 / f_{m}$ the repetition period, and $T_{0}$ the soliton pulse width. $f_{\mathrm{RF}}$ denotes the RF frequency. Note that higher-order dispersion of SMF is neglected here to give a more intuitive picture. The overall RF filter response can be seen as a periodic function of lineshape $G\left(f_{\mathrm{RF}}\right)$ with RF free spectral range (FSR) of $f_{\mathrm{FSR}}$, modulated by an envelope due to the doublesideband (DSB) modulation scheme being used. As the tap weights are all positive, the passband frequencies of the RF filters are at every multiple of $f_{\mathrm{FSR}}$, including a DC response. Throughout this article, we focus on the first passband.

Besides, by exploiting the rich soliton states of microresonator, the RF filters can be easily reconfigured at no additional cost nor complexity. Among soliton crystal structures ${ }^{38,39}$, the defect-free PSC is of particular interest, as $N\left(N \in N_{+} \mid N \geq 2\right)$ equally spaced solitons (case 2) within one round-trip time simply multiplies the initial comb spacing by $N$ times. This imparts $N$ times division of the filter passband frequencies while preserving the filter bandwidth. The automatic PSC control is equivalent to the Talbot-based processer for discrete programming the RF filters in ref. ${ }^{22}$. Less intuitively, all-optical reshaping of the RF filters can also be achieved via versatile TSM spectra (case 3 ). Two solitons residing in one period induce sinusoid interference on the sech ${ }^{2}$ spectral shape of a soliton, modulating the tap weights of the TDL filter. This rewrites the RF filter response as (see Supplementary Note 1):

$$
\begin{aligned}
H\left(f_{\mathrm{RF}}\right) \sim & \cos \left(2 \pi^{2} \phi_{2} f_{\mathrm{RF}}^{2}\right)\left[\sum_{n=-\infty}^{\infty} 2 G\left(f_{\mathrm{RF}}-n f_{\mathrm{FSR}}\right)\right. \\
& \left.+G\left(f_{\mathrm{RF}}-\left(n-\frac{\alpha}{2 \pi}\right) f_{\mathrm{FSR}}\right)+G\left(f_{\mathrm{RF}}-\left(n+\frac{\alpha}{2 \pi}\right) f_{\mathrm{FSR}}\right)\right],
\end{aligned}
$$

where $\alpha$ is the relative azimuthal angle between two solitons (expressed in radian for calculation). Clearly, new RF passbands of halved amplitude appear due to two-soliton interference, which is displaced at both sides from the initial response according to the azimuthal angle between them. Thus, the RF filter passbands can slide inside $f_{\mathrm{FSR}}$ by modifying the relative soliton angles. Unlike ref. ${ }^{16}$ in which the authors artificially introduce the sinusoidal modulation via programming the spectral carving, we alleviate the 
a
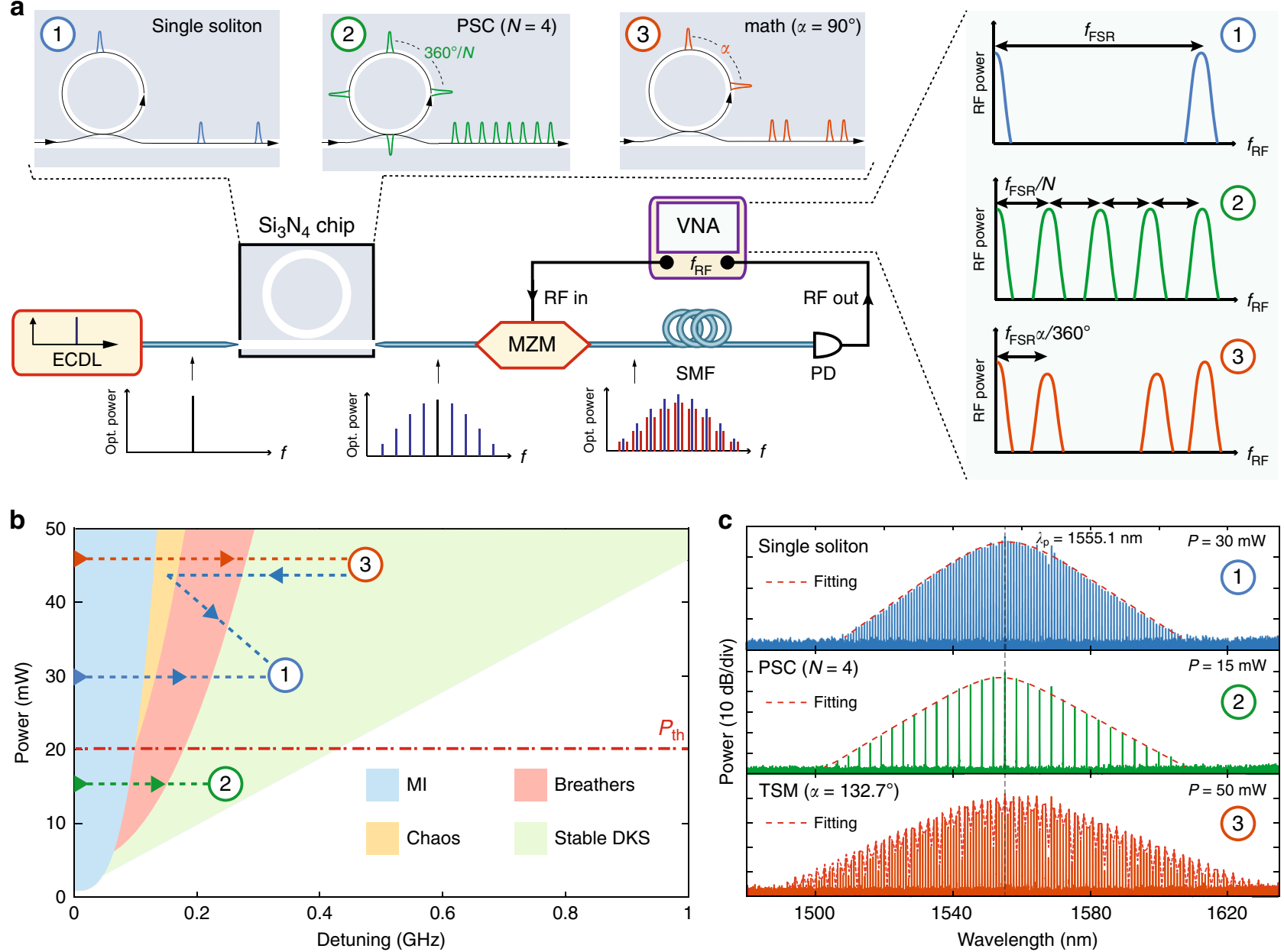

Fig. 1 Schematic diagram of reconfigurable soliton-based radiofrequency photonic filters and their underlying microcomb generation. a The conceptual setup consists of four parts: microcomb generation, radiofrequency (RF) signal upconversion, dispersive propagation, and photodetection. ECDL external cavity diode laser, MZM Mach-Zehnder modulator, SMF single-mode fiber, PD photodiode, VNA vector network analyzer. Various RF filters are synthesized based on versatile soliton microcombs: (1) single-soliton-based RF filter with a passband centered at $f_{F S R}$ (blue); (2) N - PSC- (perfect soliton crystals of $N$ equally spaced solitons within one round-trip) based RF filters with a passband centered at $f_{\mathrm{FSR}} / N$ (green, $N=4$ is shown); ( 3 ) two-soliton microcomb (TSM)-based RF filters with a passband centered at $f_{\mathrm{FSR}} \alpha / 360^{\circ}$ (orange), where $\alpha$ is the relative azimuthal angle between two solitons ( $\alpha=$ $90^{\circ}$ is shown). b Simulated stability diagram of the Lugiato-Lefever equation (LLE) involving the experimental avoided mode crossing (AMX) condition. Four different stability regions are listed: modulation instability (MI, blue), breathers (red), spatio-temporal and transient chaos (chaos, yellow), and stable dissipative Kerr soliton (DKS, green). PSC and TSM/single-soliton spectra are obtained by distinct approaches. PSC states are accessed under the threshold power to avoid the chaos region. Single-soliton or TSM states are accessed above the threshold power, by either directly falling to the states or backward tuning from a higher number of solitons. c Examples of experimentally generated spectra at resonance of 1555.1 nm: (1) single-soliton, (2) PSC $(N=4)$, and (3) $\operatorname{TSM}\left(\alpha=132.7^{\circ}\right)$ with envelope fitting. The pump power is also shown for each microcomb generation.

need for a pulse shaper and realize sinusoidal modulation by directly generating a series of TSM spectra. This novel scheme achieves wideband reconfiguration of RF filters without either interferometric configuration or additional pulse shaping.

Experimental implementation. The soliton microcombs used for RF filtering are generated from a $103.9 \mathrm{GHz}$ ultra-low loss integrated silicon nitride $\left(\mathrm{Si}_{3} \mathrm{~N}_{4}\right)$ microresonator $\left(Q \sim 1 \times 10^{7}\right)$, fabricated by the photonic Damascene reflow process ${ }^{40}$. By employing the frequency-comb-assisted diode laser spectroscopy, the detailed properties of resonances and the integrated group velocity dispersion of the microresonator are measured (see Supplementary Note 2). Strong avoided mode crossings (AMXs) are observed $\sim 1565 \mathrm{~nm}$, which lead to the modulation of intracavity CW background, thereby resulting in the ordering of the DKS pulses ${ }^{41}$ and the formation of soliton crystals ${ }^{36,38,39}$.
Figure $1 \mathrm{~b}$ shows the simulated stability diagram (see "Methods"), which consists of modulation instability (MI), breathers, chaos (spatio-temporal chaos and transient chaos), and stable DKS states. In addition, it has been revealed that the pump power level is critical for whether the PSCs or stochastic DKS states are formed $^{36}$ (see Supplementary Note 3 ). In our case, the threshold pump power $P_{\text {th }}$ is found to be $\sim 20 \mathrm{~mW}$ in the bus waveguide. When the laser scanning route is operated below threshold pump power, PSC states can be accessed without crossing the chaos region. Contrarily, DKS states with stochastic soliton number are accessed above the threshold power. Experimentally, the singlesoliton and TSM states are obtained by either directing falling to the states or backward tuning from the states with higher soliton number ${ }^{42}$. Note that switching from PSC to single soliton or TSM is prohibited unless the pump power is increased to be over the threshold ${ }^{36}$. Thus, through controlling the pump power and resonance frequency, various soliton microcombs (single soliton, 

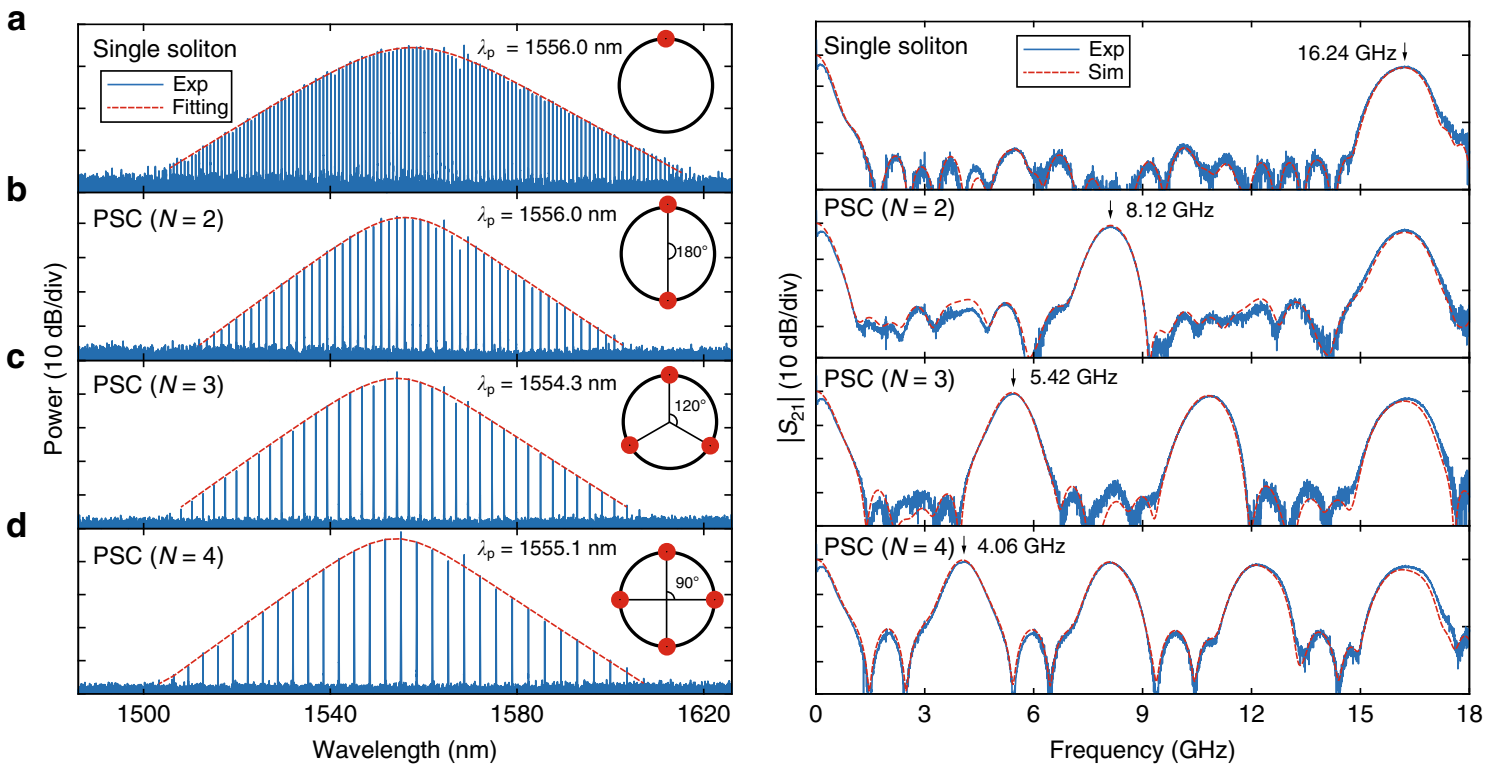

Fig. 2 Single-soliton/PSC spectra and their corresponding RF photonic filters. Through deterministic accessing PSC states of different resonances, the RF filter passbands can be divided correspondingly. Left column: microcomb spectra (blue: experiment; red: sech ${ }^{2}$ fitting); right column: corresponding normalized RF filter responses (blue: experiment; red: simulation). a RF filter centered at $16.24 \mathrm{GHz}$ based on single soliton from resonance of $1556.0 \mathrm{~nm}$; b-d RF filters centered at $8.12,5.42$, and $4.06 \mathrm{GHz}$ based on two, three, four times PSC, generated at resonances of 1556.0, 1554.3, and 1555.1 nm, respectively. The insets of left column illustrate soliton distribution inside the microresonator: a single-soliton; b-d equally spaced solitons (PSCs) with adjacent angles of $180^{\circ}, 120^{\circ}$, and $90^{\circ}$, respectively $\left(360^{\circ} / N, N=2,3,4\right)$.

PSC, and TSM) can be obtained on demand to produce the desired RF filter responses. For example, Fig. 1c shows three distinct optical spectra obtained from the resonance of $1555.1 \mathrm{~nm}$ : single soliton, PSC $(N=4)$, and TSM $\left(\alpha=132.7^{\circ}\right.$, see "Methods"), respectively. It is also worthwhile to mention that although this type of RF filter does not require coherent comb states, the high-intensity noise of MI combs is certainly undesirable ${ }^{15}$. As the intensity noise will be transferred to the synthesized RF filters, mode-locked comb states are preferred to minimize the noise at the RF output.

Figure 2 depicts the RF photonic filters using single soliton and various PSC microcombs. The single-soliton-based RF filter (Fig. 2a) is centered at $16.24 \mathrm{GHz}$, with main-to-sidelobe suppression ratio (MSSR) of $23.2 \mathrm{~dB}$. Further, various PSC states are deterministically obtained at different resonances under the threshold power, thereby all optically reconfiguring the corresponding RF filters. The comb spacing multiplication via PSC results in the division of the corresponding RF passbands. RF filters centered at $8.12,5.42$, and $4.06 \mathrm{GHz}$ (Fig. 2b-d) are experimentally synthesized through 2,3 , and 4 equally spaced solitons, with MSSR of 22.6, 25.6, and $20.4 \mathrm{~dB}$, respectively. All these RF filters achieve MSSR over $20 \mathrm{~dB}$ without additional programmable spectral shaping. The MSSR here is limited by the smoothness of the optical spectra ${ }^{19}$, as several AMX can be seen in the microcombs. Nevertheless, all these microcombs preserve well the sech ${ }^{2}$ envelope, and remained smooth after amplification. In addition, the measured RF filter responses are in excellent agreement with simulations, by taking into account of third-order dispersion $\left(\beta_{3}\right)$ of SMF (see "Methods"). Note that the bandwidths of experimentally synthesized RF filters broaden slightly with their center frequencies, also due to the third-order dispersion of SMF ${ }^{15}$.

Figure $3 \mathrm{a}$ shows the TSM spectra and their corresponding RF filter responses, pumped at resonance of $1556.0 \mathrm{~nm}$. According to Eq. (2), the first passband frequencies of RF filters scale linearly with the relative angles between two solitons, so that the filter reconfiguration is achieved. In the experiment, TSM spectra with relative angles of $19.7^{\circ}, 43.0^{\circ}, 68.1^{\circ}, 94.6^{\circ}, 117.0^{\circ}, 142.5^{\circ}$, and $169.2^{\circ}$ are obtained, where the angles are extracted from the fitting of the microcomb spectral envelope (see "Methods"). The measured RF filters are correspondingly centered at $0.85,1.96$, $3.05,4.24,5.26,6.40$, and $7.51 \mathrm{GHz}$, confirming the linear relation with the soliton angle (see Supplementary Note 4). As in the case of PSC, a slight broadening of the filter passband width from 490 to $620 \mathrm{MHz}$ is attributed to the third-order dispersion of SMF. Overall, the RF filters obtained at resonance $1556.0 \mathrm{~nm}$ could vary from $\mathrm{DC}$ to $8.1 \mathrm{GHz}\left(f_{\mathrm{FSR}} / 2\right)$ with a maximum grid of $1.2 \mathrm{GHz}$, while roughly preserving the filter bandwidth in the meantime. The granularity of TSM-based RF filters can be further reduced to $<1 \mathrm{GHz}$ by exploiting adjacent resonances of $1556.0 \mathrm{~nm}$ (see Supplementary Note 4). Also, mirrored passband responses of the TSM-based RF filters coexist between 8.1 and $16.2 \mathrm{GHz}$.

Importantly, the possible angles between two solitons are determined by the overall AMX profile, and are rather robust to both laser power and frequency detuning, thereby deterministically dictating the filter passband frequencies to be either one of those shown in Fig. 3a. To gain insights into the relative angles between two solitons, we also perform perturbed Lugiato-Lefever equation (LLE) simulation to investigate the TSM formations (see "Methods"). The blue curve in Fig. 3b shows one example of the steady-state two-soliton temporal intracavity profile. Due to the AMX effect, periodic intensity modulation is observed upon the CW background. It is clearly seen that the soliton can only be excited at specific parameter gradients ${ }^{41}$, as manifested by the green dashed lines, which correspond to the stationary solutions obtained in the simulation. These possible soliton angles are in good agreement with the experimental results, which is indicated as red dashed lines. To further test the robustness of the angle between two solitons, an external perturbation is deliberately introduced on their relative angle. Figure $3 \mathrm{c}$ illustrates the 
a

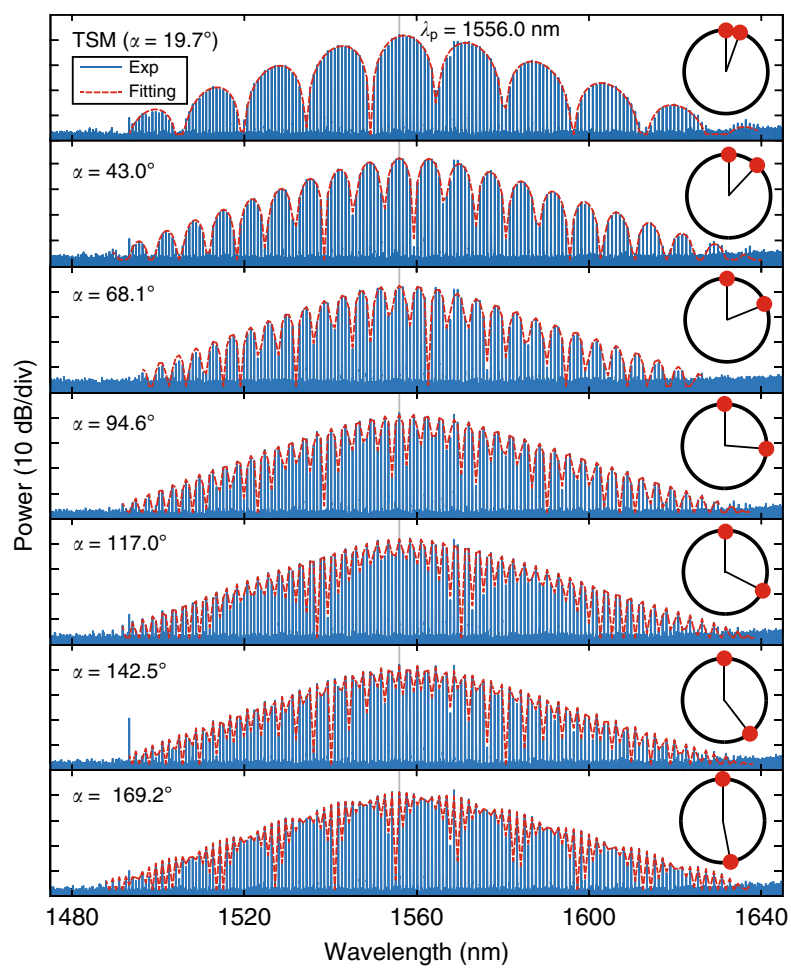

b

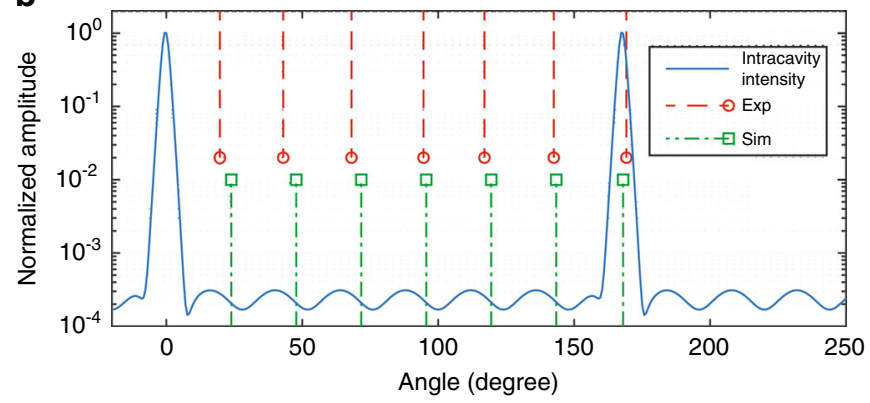

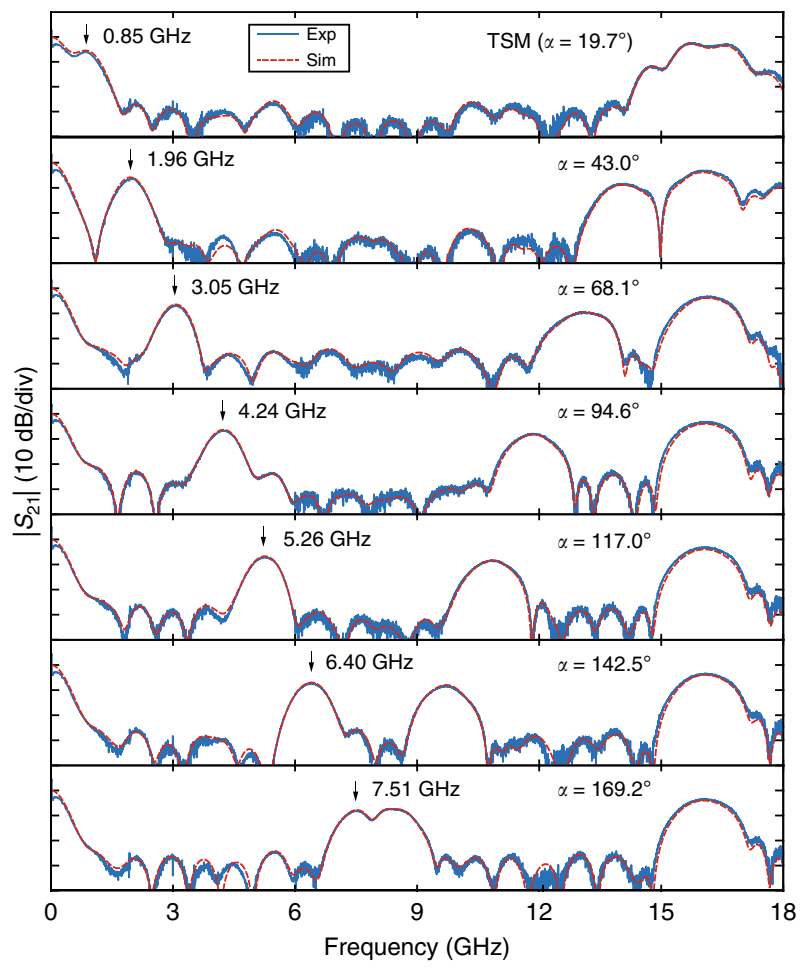

c

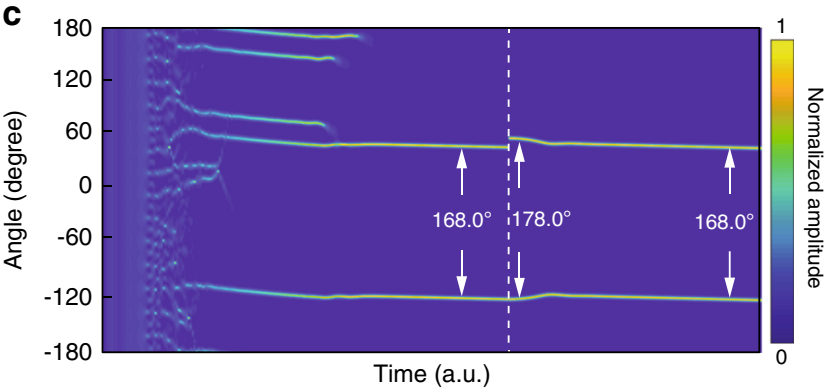

Fig. 3 TSM spectra and their corresponding RF photonic filters, together with TSM simulation investigation. By accessing different two-soliton states, the RF filters can be all optically reconfigured. a Left column: TSM spectra at resonance of $1556.0 \mathrm{~nm}$ (blue: experiment; red: envelope fitting). The insets

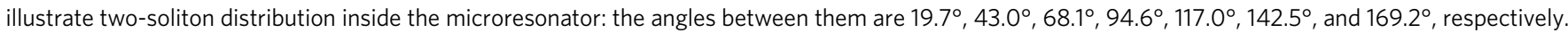
Right column: corresponding normalized RF filter responses (blue: experiment; red: simulation) with passbands at 0.85, 1.96, 3.05, 4.24, 5.26, 6.40, and $7.51 \mathrm{GHz}$, respectively. b Simulation of TSM relative azimuthal angles. One example of the simulated TSM intracavity intensity profile (blue), where AMXinduced background modulation is observed. The red and green lines, respectively, indicate the measured and simulated possible azimuthal angles between two solitons. c Simulation of the intracavity waveform evolution of TSM for robustness test. First, TSM state with a relative angle of $168.0^{\circ}$ is excited by scanning the pump over the resonance. Once the TSM becomes stable, a $10.0^{\circ}$ perturbation is introduced to one of the solitons at white dashed line. The relative angle will re-stabilize to the original angle of $168.0^{\circ}$ after a period of free running.

dynamical evolution of the two-soliton formation. The simulation is initiated as a standard laser scanning scheme to kick out two solitons. Once the simulation reaches a stable two-soliton solution (relative angle of $168.0^{\circ}$ ), one of the solitons is dragged from its original position by a $10.0^{\circ}$ on purpose. After a period of free-running, the two solitons converge back to their original relative positions, again at $168.0^{\circ}$ apart. This confirms the regulation of two solitons under AMX background modulation.

RF filter reconfiguration experiment. A proof-of-concept $R F$ filter reconfiguration experiment is also illustrated in Fig. 4 using TSM-based RF filters (see "Methods"). Two superimposed phaseshift keying (PSK) signals in which a $40 \mathrm{Mb} / \mathrm{s}$ modulation at 1.96 $\mathrm{GHz}$ tone and a $20 \mathrm{Mb} / \mathrm{s}$ modulation at $3.05 \mathrm{GHz}$ tone are prepared as input test signals. The RF filters are then, respectively, reconfigured at 1.96 and $3.05 \mathrm{GHz}$ to filter the input signals, by triggering the TSM spectra of corresponding soliton angles. At the output of the RF filters, the nearly complete rejection of either one of the PSK signals is observed on the electrical spectra (Fig. 4a), where the extinction ratios exceed $35 \mathrm{~dB}$ for both cases. Figure $4 \mathrm{~b}$, c shows the filtered output RF waveforms. The periodicity of the output temporal traces corroborates the filtering of the original RF signals.

\section{Discussion}

A comparison of the soliton RF filters presented in this work with other RF photonic filters based on various multiwavelength sources is shown in Table 1. First, we see that the MSSR achieved by soliton microcomb is comparable or even better than other implementations that do not require pulse shapers, such as combs 

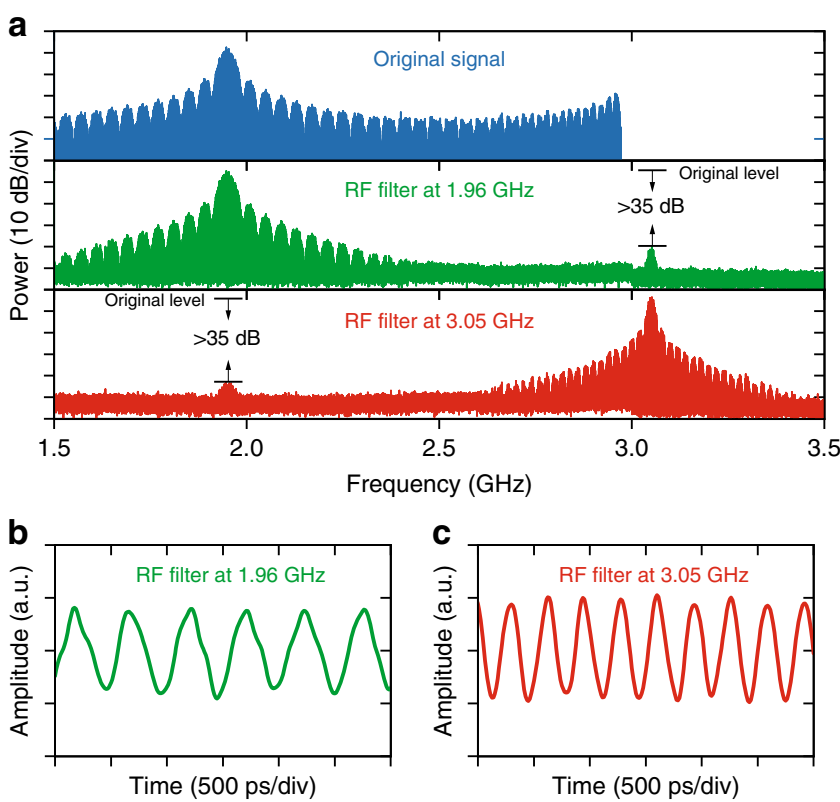

Fig. 4 Proof-of-concept experiment using TSM-based RF filters. Two PSK signals with $40 \mathrm{Mb} / \mathrm{s}$ modulation at $1.96 \mathrm{GHz}$ and $20 \mathrm{Mb} / \mathrm{s}$ modulation at $3.05 \mathrm{GHz}$ are filtered by the TSM-based RF filters centered at 1.96 and 3.05 $\mathrm{GHz}$, respectively. a From top to bottom: electrical spectra of original microwave signal, signal after $1.96 \mathrm{GHz}$ filter, and signal after $3.05 \mathrm{GHz}$ filter. b Waveform after $1.96 \mathrm{GHz}$ filter. c Waveform after $3.05 \mathrm{GHz}$ filter.

based on cascaded modulators ${ }^{19}$, laser arrays ${ }^{7}$, or mode-locked lasers ${ }^{21,22}$, while none of them is integrated solutions regarding the light sources. Second, all of the past microcomb-based RF filters require, to our knowledge, two high-fidelity pulse shapers ${ }^{15-17}$, in order to equalize the largely unbalanced comb line intensity in the complex microcomb states. The achievable MSSR is indeed higher as expected, due to the fine equalization of comb amplitudes. Without pulse shapers, these approaches are not able to achieve similar MSSR nor tunability. In our case, the MSSR is mainly limited by the spectral roughness induced by mode crossings. One could envision combining our current implementation with an integrated spectral shaper as previously demonstrated $^{8}$, although the device is based on indium phosphide platform. The integrated shaper can enhance our filters with improved MSSR and faster reconfiguration, while the soliton microcombs can greatly simplify the EO combs incorporating seven cascaded modulators used in ref. ${ }^{8}$. It is worth noting that using integrated shaper for comb equalization in complex microcomb spectra would be inefficient, due to the shaper's limited extinction ratio. Thus, the ability to control the soliton states among microcombs is truly essential for practical implementation.

From the table, it is also evident that only a few past works report the link performances of comb-based RF filters. We carried out simple optimization of the RF filter link specifically based on 4-PSC as an example, where we achieve simultaneous positive link gain and noise figure within $30 \mathrm{~dB}$ (see Supplementary Note 5). Although the link performance is not yet as good as that of pure microwave solutions, it is already very close to the optimized performances of the state-of-the-art comb-based RF filters $^{43}$. In addition, we achieve widely reconfigurable RF photonic filters from 0.8 to $16.2 \mathrm{GHz}$, taking into account both passbands obtained from TSM spectra. This multioctave filter operation is generally considered challenging by pure RF engineering ${ }^{44,45}$, especially at higher carrier frequency towards millimeter wave or

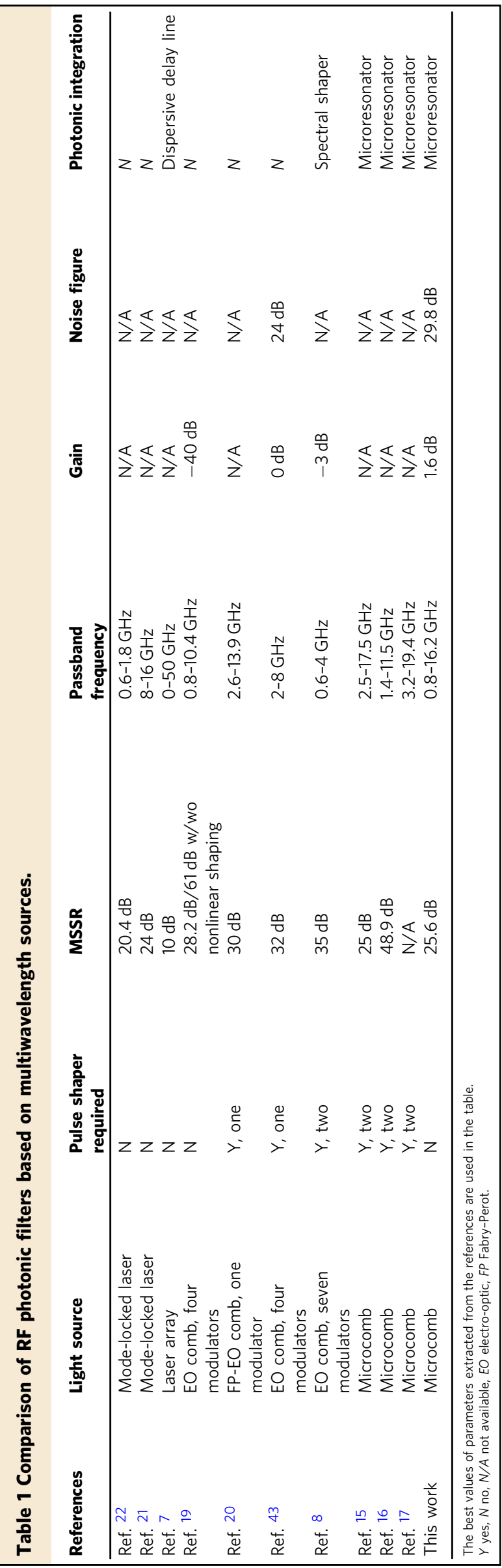


terahertz wave. However, there is no such limitation for microwave photonic filters. It is worth mentioning the recent work on RF photonic filters synthesized from passive cascaded ring resonators with a wide continuous tuning capability ${ }^{12}$. However, in order to configure the filters, a number of power-consuming thermal heaters have to be controlled with fine resolution voltage sources. Although our filters cannot be tuned continuously, the exploration of multiple resonances dramatically enriches the achievable RF filters, while the possible controlled mode interaction ${ }^{46}$ or using dichromatic pumps ${ }^{47}$ may empower our filters with continuous tuning ability (see Supplementary Note 6). Moreover, additional functionalities, such as photonic downconversion of microwave signals, can be simultaneously realized in the microcomb-based filter setup, without electrical mixer and local oscillators ${ }^{48}$. The microcomb FSR will act as the local oscillator, which is promising for on-chip terahertz wave signal processing.

The RF filters obtained in this study are based on microcombs generated in a $103.9 \mathrm{GHz}$ microresonator. If one targets RF filters with narrower bandwidth, a microresonator with larger size generating smaller FSR comb will be a better choice, as the filter bandwidth scales with the comb FSR given a certain soliton pulse width (see Supplementary Note 1). The recent ultra-low loss $\mathrm{Si}_{3} \mathrm{~N}_{4}$ technology has allowed soliton formation down to the 10 $\mathrm{GHz}$ range ${ }^{35}$, which would enable RF filters of only tens of $\mathrm{MHz}$ bandwidth without additional pulse shaping. However, in order to maintain the filter center frequency when using small FSR combs, longer dispersive fiber is needed, while the envelope fading becomes more severe under the DSB modulation scheme ${ }^{20}$. In this case, more sophisticated modulation schemes are required, such as single-sideband modulation with broadband electrical $90^{\circ}$ hybrid, in order to eliminate the fading effect. In comparison, microcombs of large comb spacing allow for a simple RF uploading method. Also, since we are interested in RF filters up to $\sim 16 \mathrm{GHz}$ (limited by the measurement range of VNA), the underlying combs with repetition rate over $32 \mathrm{GHz}$ are preferred to avoid spurs generated from the signal beating with other comb lines ${ }^{19}$. Since obtaining a narrow RF photonic filter is not the primary interest in the current study, we utilize the 103.9 $\mathrm{GHz}$ microresonator in the experiment. The techniques presented here are compatible with other microcomb FSR.

In summary, we demonstrate reconfigurable soliton-based RF photonic filters using simplified approaches. Contrary to previous demonstrations where pulse shapers are necessary to obtain descent passband responses ${ }^{15-17}$, the proposed schemes are intrinsically well shaped with the smooth spectral envelopes of solitons. More importantly, we harness various intrinsic DKS states of microresonator, like PSC and TSM, for RF filter reconfiguration at no additional cost. The diversity and regularization of soliton formats in microresonator are investigated in the favor of RF photonic filters. To a certain extent, these inherent soliton states could be in place of substantial efforts made in the past for reconfiguring the comb-based RF filters, such as using interferometric architecture ${ }^{15,19}$, programmable pulse shaping ${ }^{16,20}$, or Talbot-based signal processor 22 . Although our soliton-based RF filters are much simpler than previous microcomb-based RF filters $^{15-17}$ as we eliminate the use of two bulky pulse shapers, the form factor is not yet as compact as their electrical counterparts. Nevertheless, the basic components of our filters can be integrated $^{7}$. The recent advancements on the integration between laser chip and microresonator ${ }^{28,29}$, as well the possibility to replace the SMF with a highly dispersive integrated waveguide ${ }^{7}$, can be further connected to the current work for miniaturization. To conclude, our work significantly reduces the system complexity, size, and cost of the microcomb-based RF filters, while preserving their wide reconfigurability. The proposed schemes set as a stepping stone for chip-scale, cost-effective, and widely reconfigurable microcomb-based RF filters.

\section{Methods}

Experimental setup. A C-band tunable CW laser is amplified by an Erbium-doped fiber amplifier (EDFA) with amplified spontaneous emission (ASE) filtered, polarization aligned at the TE mode, and then coupled to the $\mathrm{Si}_{3} \mathrm{~N}_{4}$ microresonator for soliton microcomb generation. The input and output coupling of the chip is achieved via lensed fibers of $\sim 30 \%$ fiber-chip-fiber coupling efficiency. The soliton microcombs are initiated by scanning the pump over the resonances, with the assistance of an arbitrary function generator ${ }^{27}$. The residual pump of generated microcombs is then filtered by a tunable fiber Bragg grating, while a circulator is inserted in between to avoid back-reflection. $10 \%$ of light is tapped to an optical spectrum analyzer to record the microcomb spectra. The other $90 \%$ of the light is amplified, and polarization managed, before sending to a $30 \mathrm{GHz}$ bandwidth MZM. RF signals from the VNA are applied to the MZM in DSB modulation format. The modulated spectra are then propagated through a spool of $4583.8 \mathrm{~m}$ SMF to acquire dispersive delays, and finally beats at an $18 \mathrm{GHz}$ PD to convert the signals back to the RF domain. The length of SMF is measured by a commercial optical time-domain reflectometer.

For the system demonstration, a $12 \mathrm{GSa} / \mathrm{s}$ arbitrary waveform generator (AWG) is used to prepare the input RF signals. A $40 \mathrm{Mb} / \mathrm{s}$ PSK signal modulated at $1.96 \mathrm{GHz}$ tone and $20 \mathrm{Mb} / \mathrm{s}$ PSK signal modulated at $3.05 \mathrm{GHz}$ tone are generated separately from the two channels of the AWG. After adding the two streams of signals in a combiner, the composite signal is then sent through the TSM-based RF filters, tuned at 1.96 and $3.05 \mathrm{GHz}$, respectively. The output spectra are measured by an electrical spectrum analyzer, while the waveforms are measured using a highspeed real-time oscilloscope.

$\mathbf{S i}_{3} \mathbf{N}_{\mathbf{4}}$ microresonator. The $\mathrm{Si}_{3} \mathrm{~N}_{4}$ microresonator used in the experiment is a ring structure with a radius of $217 \mu \mathrm{m}$. Its waveguide cross-section (width $\times$ height) is made to be $1500 \mathrm{~nm} \times 750 \mathrm{~nm}$. The microresonator is coupled with a bus waveguide, which possesses the same cross-section as the ring to realize high coupling ideality ${ }^{40}$. To achieve critical coupling for the resonances, the gap distance between the ring and bus waveguide is designed to be $690 \mathrm{~nm}$. In our experiment, the pumped resonances are around $1556 \mathrm{~nm}$, where both the intrinsic linewidths and coupling strengths are approximately $20 \mathrm{MHz}$ (see Supplementary Note 2). With respect to the reference resonance of $\omega_{0} / 2 \pi=192.8 \mathrm{THz}$, the dispersion parameters of microresonator are measured: FSR of microresonator $D_{1} / 2 \pi \approx 103.9 \mathrm{GHz}$, second-order dispersion term $D_{2} / 2 \pi \approx 1.28 \mathrm{MHz}$, and negligible third-order dispersion term $D_{3} / 2 \pi \sim \mathcal{O}(1) \mathrm{kHz}$ (see Supplementary Note 2).

LLE simulation. The simulation performed in this work is based on the perturbed LLE model:

$$
\frac{\partial A(\phi, t)}{\partial t}=-\left(\frac{\kappa}{2}+j\left(\omega_{0}-\omega_{p}\right)\right) A(\phi, t)+j \frac{D_{2}}{2} \frac{\partial^{2} A(\phi, t)}{\partial \phi^{2}}+j g|A(\phi, t)|^{2} A(\phi, t)+\sqrt{\kappa_{\mathrm{ex}}} s_{\mathrm{in}},
$$

where $A(\phi, t)$ is the temporal envelope of the intracavity field. $\kappa=\kappa_{\mathrm{ex}}+\kappa_{0}$ is the total cavity loss rate, where $\kappa_{\mathrm{ex}}$ is the coupling rate, and $\kappa_{0}$ is the internal loss rate $\omega_{0}$ and $\omega_{\mathrm{p}}$ denote the angular frequencies of the pumped resonance and the CW pump laser, respectively. $g$ is the Kerr frequency shift per photon, defined as $g=\hbar \omega_{0}^{2} c n_{2} / n_{0}^{2} V_{\text {eff }}$, where $n_{0}$ is the effective group refractive index, $n_{2}$ is the nonlinear optical index, and $V_{\text {eff }}$ is the effective mode volume. $D_{2}$ corresponds to the second-order dispersion term, and $\left|s_{\text {in }}\right|^{2}$ is the pump power. To involve the AMX effect, an additional frequency detuning $\Delta_{k}$ is introduced at the $k$-th mode, so that the frequency of the $k$-th mode becomes $\omega_{k}=\omega_{0}+D_{1} k+D_{2} k^{2} / 2+\Delta_{k}$ (see Supplementary Note 2). Here in simulation, the dispersion is limited to $D_{2}$, and the Raman and thermal effects are not taken into account. According to the dispersion measurement and the generated microcomb spectra, the parameters for the AMX in the simulation are set as $k=15$ and $\Delta_{k} / 2 \pi=100 \mathrm{MHz}$, enabling the modulation of the CW intracavity background for the trapping of soliton temporal positions. Note that the strength of the AMX here is estimated to introduce the regularizability of solitons, but without disturbing their formations ${ }^{36}$. Other parameters used in the simulation are retrieved from the characterization, that is, $D_{1} / 2 \pi=$ 103.9 GHz, $D_{2} / 2 \pi=1.28 \mathrm{MHz}, \kappa_{\mathrm{ex}} / 2 \pi=\kappa_{0} / 2 \pi=\kappa / 4 \pi=20 \mathrm{MHz}$.

The simulation of the stability chart is obtained by initializing the numerical model with single-soliton solution at various pump power and detuning conditions $^{36}$. Four different states are found: MI, breathers, spatio-temporal and transient chaos, and stable DKS states. The threshold pump power, separating the PSC and stochastic DKS formations, is estimated from both the simulation and experimental conditions. For the TSM simulation, the numerical model is operated under standard CW laser pump scanning from blue-detuned to red-detuned side, until it reaches the stable TSM states. All the possible angles of TSM are recorded. To test the robustness of the TSM azimuthal angle, the model is initialized with one of the exact two-soliton solution, but deliberately perturbed by $10.0^{\circ}$ angle deviation. Two solitons are gradually re-stabilized at its original azimuthal angle after a period of free running. 
RF filter response fitting. As the generated microcomb spectra are broader than the amplifying bandwidth of EDFA, we also measured the optical spectra after the EDFA, in order to extract the TDL filter tap weights. The third-order dispersion $\beta_{3}$ of SMF is taken into account for the fitting of RF responses, which can be formulated as ${ }^{20}$.

$$
\begin{aligned}
H\left(f_{\mathrm{RF}}\right) \sim & \sum_{k} p_{k} \cos \left(2 \pi^{2} \phi_{2} f_{\mathrm{RF}}^{2}+4 \pi^{3} \phi_{3} k f_{m}^{2} f_{\mathrm{RF}}\right) \exp \left(j 4 \pi^{2} \phi_{2} k f_{m} f_{\mathrm{RF}}\right. \\
& \left.+j 4 \pi^{3} \phi_{3} k^{2} f_{m}^{2} f_{\mathrm{RF}}+j \frac{4}{3} \pi^{3} \phi_{3} f_{m}^{3}\right),
\end{aligned}
$$

where $\phi_{3}=-\beta_{3} L$. In accordance with typical values of SMF dispersion, $\beta_{2}=-20.2$ $\mathrm{ps}^{2} / \mathrm{km}$ and $\beta_{3}=0.117 \mathrm{ps}^{3} / \mathrm{km}$ at $1550 \mathrm{~nm}$ are estimated for all the above fittings of RF filters. The simulation results are in excellent agreement with experimental RF filter responses.

TSM spectral fitting. First, we extract the power of each comb mode of experimental TSM spectra, and indexed them with respect to the pump mode. Pump mode is rejected and amplitude rescaling is considered as a fitting parameter. Note that the amount of spectral redshift due to Raman effect is also estimated in fitting, by displacing the center of sech ${ }^{2}$ soliton spectra from the pump comb mode. Then, the rescaling parameter and redshift are estimated to best fit the experimental data with the TSM spectral power equation (see Supplementary Note 1), thereby retrieving the azimuthal angle $\alpha$ between two solitons. Excellent match between simulations and experimental spectra are obtained.

\section{Data availability}

The data used to produce the results of this paper are available at https://doi.org/10.5281/ zenodo. 3902646 .

\section{Code availability}

The code used to produce the results of this paper is available at https://doi.org/10.5281/ zenodo. 3902646 .

Received: 2 March 2020; Accepted: 7 August 2020;

Published online: 01 September 2020

\section{References}

1. Capmany, J. \& Novak, D. Microwave photonics combines two worlds. Nat. Photonics 1, 319 (2007).

2. Marpaung, D., Yao, J. \& Capmany, J. Integrated microwave photonics. Nat. Photonics 13, 80 (2019).

3. Yao, J. Microwave photonics. J. Lightwave Technol. 27, 314-335 (2009).

4. Marpaung, D. et al. Integrated microwave photonics. Laser Photonics Rev. 7, 506-538 (2013).

5. Wu, J. et al. Rf photonics: an optical microcombs' perspective. IEEE J. Sel. Top. Quantum Electron. 24, 1-20 (2018).

6. Xie, Y. et al. Programmable optical processor chips: toward photonic rf filters with dsp-level flexibility and mhz-band selectivity. Nanophotonics 7, 421-454 (2017).

7. Sancho, J. et al. Integrable microwave filter based on a photonic crystal delay line. Nat. Commun. 3, 1075 (2012)

8. Metcalf, A. J. et al. Integrated line-by-line optical pulse shaper for high-fidelity and rapidly reconfigurable rf-filtering. Opt. Express 24, 23925-23940 (2016).

9. Zhuang, L., Roeloffzen, C. G., Hoekman, M., Boller, K.-J. \& Lowery, A. J. Programmable photonic signal processor chip for radiofrequency applications. Optica 2, 854-859 (2015).

10. Marpaung, D. et al. Si 3 n 4 ring resonator-based microwave photonic notch filter with an ultrahigh peak rejection. Opt. Express 21, 23286-23294 (2013).

11. Pan, S., Tang, Z., Huang, M. \& Li, S. Reflective-type microring resonator for on-chip reconfigurable microwave photonic systems. IEEE J. Sel. Top. Quant. Electron. 26, https://doi.org/10.1109/JSTQE.2020.2969567 (2020).

12. Jiang, H., Yan, L. \& Marpaung, D. Chip-based arbitrary radio-frequency photonic filter with algorithm-driven reconfigurable resolution. Opt. Lett. 43, 415-418 (2018).

13. Eggleton, B. J., Poulton, C. G., Rakich, P. T., Steel, M. J. \& Bahl, G. Brillouin integrated photonics. Nat. Photonics 13, 1-14 (2019).

14. Fandiño, J. S., Muñoz, P., Doménech, D. \& Capmany, J. A monolithic integrated photonic microwave filter. Nat. Photonics 11, 124 (2017).

15. Xue, X. et al. Programmable single-bandpass photonic rf filter based on kerr comb from a microring. J. Lightwave Technol. 32, 3557-3565 (2014).

16. Xu, X. et al. Advanced adaptive photonic rf filters with 80 taps based on an integrated optical micro-comb source. J. Lightwave Technol. 37, 1288-1295 (2019).

17. $\mathrm{Xu}, \mathrm{X}$. et al. High performance rf filters via bandwidth scaling with kerr microcombs. APL Photonics 4, 026102 (2019).
18. Capmany, J., Ortega, B. \& Pastor, D. A tutorial on microwave photonic filters J. Lightwave Technol. 24, 201-229 (2006)

19. Supradeepa, V. et al. Comb-based radiofrequency photonic filters with rapid tunability and high selectivity. Nat. Photonics 6, 186 (2012).

20. Zhu, X., Chen, F., Peng, H. \& Chen, Z. Novel programmable microwave photonic filter with arbitrary filtering shape and linear phase. Opt. Express 25, 9232-9243 (2017).

21. Ortigosa-Blanch, A., Mora, J., Capmany, J., Ortega, B. \& Pastor, D. Tunable radio-frequency photonic filter based on an actively mode-locked fiber laser. Opt. Lett. 31, 709-711 (2006).

22. Maram, R., Onori, D., Azaña, J. \& Chen, L. R. Discretely programmable microwave photonic filter based on temporal talbot effects. Opt. Express 27, 14381-14391 (2019).

23. Xue, X. et al. Microcomb-based true-time-delay network for microwave beamforming with arbitrary beam pattern control. J. Lightwave Technol. $\mathbf{3 6}$ 2312-2321 (2018).

24. $\mathrm{Xu}, \mathrm{X}$. et al. Broadband $\mathrm{rf}$ channelizer based on an integrated optical frequency kerr comb source. J. Lightwave Technol. 36, 4519-4526 (2018).

25. Tan, M. et al. Microwave and rf photonic fractional hilbert transformer based on a 50 ghz kerr micro-comb. J. Lightwave Technol. 37, 6097-6104 (2019).

26. Xue, X. et al. Mode-locked dark pulse kerr combs in normal-dispersion microresonators. Nat. Photonics 9, 594 (2015).

27. Herr, T. et al. Temporal solitons in optical microresonators. Nat. Photonics $\mathbf{8}$ 145 (2014).

28. Stern, B., Ji, X., Okawachi, Y., Gaeta, A. L. \& Lipson, M. Battery-operated integrated frequency comb generator. Nature 562, 401 (2018).

29. Raja, A. S. et al. Electrically pumped photonic integrated soliton microcomb Nat. Commun. 10, 680 (2019).

30. Marin-Palomo, P. et al. Microresonator-based solitons for massively parallel coherent optical communications. Nature 546, 274 (2017).

31. Suh, M.-G. \& Vahala, K. J. Soliton microcomb range measurement. Science 359, 884-887 (2018).

32. Wang, J. et al. Long distance measurement using single soliton microcomb. Preprint at http://arXiv.org/abs/2002.10565 (2020).

33. Suh, M.-G., Yang, Q.-F., Yang, K. Y., Yi, X. \& Vahala, K. J. Microresonator soliton dual-comb spectroscopy. Science 354, 600-603 (2016).

34. Obrzud, E. et al. A microphotonic astrocomb. Nat. Photonics 13, 31 (2019).

35. Liu, J. et al. Photonic microwave generation in the $\mathrm{x}$-and $\mathrm{k}$-band using integrated soliton microcombs. Nat. Photonics 14, 486-491 (2020).

36. Karpov, M. et al. Dynamics of soliton crystals in optical microresonators. Nat Phys. https://doi.org/10.1038/s41567-019-0635-0, 1-7 (2019).

37. He, Y., Ling, J., Li, M. \& Lin, Q. Perfect soliton crystals on demand. Laser Photonics Rev. 14, 1900339 (2020)

38. Cole, D. C., Lamb, E. S., Del'Haye, P., Diddams, S. A. \& Papp, S. B. Soliton crystals in kerr resonators. Nat. Photonics 11, 671 (2017).

39. Wang, W. et al. Robust soliton crystals in a thermally controlled microresonator. Opt. Lett. 43, 2002-2005 (2018).

40. Liu, J. et al. Ultralow-power chip-based soliton microcombs for photonic integration. Optica 5, 1347-1353 (2018).

41. Wang, Y. et al. Universal mechanism for the binding of temporal cavity solitons. Optica 4, 855-863 (2017).

42. Guo, H. et al. Universal dynamics and deterministic switching of dissipative kerr solitons in optical microresonators. Nat. Phys. 13, 94 (2017).

43. Kim, H.-J., Leaird, D. E., Metcalf, A. J. \& Weiner, A. M. Comb-based rf photonic filters based on interferometric configuration and balanced detection. J. Lightwave Technol. 32, 3478-3488 (2014).

44. Long, J., Li, C., Cui, W., Huangfu, J. \& Ran, L. A tunable microstrip bandpass filter with two independently adjustable transmission zeros. IEEE Microw. Wirel. Compon. Lett. 21, 74-76 (2011).

45. Lee, B., Nam, S., Koh, B., Kwak, C. \& Lee, J. K-band frequency tunable substrate-integrated-waveguide resonator filter with enhanced stopband attenuation. IEEE Trans. Microw. Theory Tech. 63, 3632-3640 (2015).

46. Xue, X. et al. Normal-dispersion microcombs enabled by controllable mode interactions. Laser Photonics Rev. 9, L23-L28 (2015).

47. Taheri, H., Matsko, A. B. \& Maleki, L. Optical lattice trap for kerr solitons. Eur. Phys. J. D 71, 153 (2017).

48. Torres-Company, V., Leaird, D. E. \& Weiner, A. M. Simultaneous broadband microwave downconversion and programmable complex filtering by optical frequency comb shaping. Opt. Lett. 37, 3993-3995 (2012).

\section{Acknowledgements}

This work was supported by the Swiss National Science Foundation under Grant Agreement Nos. 159897, 176563 (BRIDGE), and 192293, by the Hasler Foundation, by contract FA9550-19-C-7001 (KECOMO) from the Defense Advanced Research Projects Agency (DARPA), Microsystems Technology Office (MTO), and by the Air Force Office of Scientific Research, Air Force Materiel Command, USAF under Award No. FA9550-19-10250. The samples were fabricated in the EPFL Center of MicroNanoTechnology (CMi). 


\section{Author contributions}

J.Hu and J.He conceived the idea. J.Hu, J.He, A.S.R., and J.L. performed the experiments. J.L. fabricated the devices. T.J.K. supervised the sample fabrication and comb generation. J.He and J.Hu carried out the theoretical analysis and numerical simulations, with the assistance of M.K. and A.L. C.-S.B. supervised the project. All authors contributed to the writing of the manuscript.

\section{Competing interests}

The authors declare no competing interests.

\section{Additional information}

Supplementary information is available for this paper at https://doi.org/10.1038/s41467020-18215-z.

Correspondence and requests for materials should be addressed to T.J.K. or C.-S.B.

Peer review information Nature Communications thanks Leimeng Zhuang and the other, anonymous, reviewer(s) for their contribution to the peer review of this work.
Reprints and permission information is available at http://www.nature.com/reprint

Publisher's note Springer Nature remains neutral with regard to jurisdictional claims in published maps and institutional affiliations.

(c) Open Access This article is licensed under a Creative Commons Attribution 4.0 International License, which permits use, sharing, adaptation, distribution and reproduction in any medium or format, as long as you give appropriate credit to the original author(s) and the source, provide a link to the Creative Commons license, and indicate if changes were made. The images or other third party material in this article are included in the article's Creative Commons license, unless indicated otherwise in a credit line to the material. If material is not included in the article's Creative Commons license and your intended use is not permitted by statutory regulation or exceeds the permitted use, you will need to obtain permission directly from the copyright holder. To view a copy of this license, visit http://creativecommons.org/ licenses/by/4.0/.

(C) The Author(s) 2020 